\title{
Heilkundeübertragung - Modellvorhaben oder schon Realität?!
}

\author{
Antje Koeppe
}

Online publiziert: 24. Oktober 2012

(C) Springer-Verlag Wien 2012

Für die Pflegenden in vielen Krankenhäusern (überwiegend kleine Häuser der Basisversorgung in ländlichen Regionen) wurde die Richtlinie zu Modellvorhaben schon von der Praxis eingeholt. Derzeitig ist es so, dass viele Stellen des ärztlichen Dienstes unbesetzt sind und aus unterschiedlichen Gründen nicht nachbesetzt werden können. Um jedoch Betriebsabläufe patientenorientiert zu sichern, Verweildauern einzuhalten, Wartezeiten zu verkürzen und letztendlich Erlöse zu sichern, müssen viele ärztliche Tätigkeiten schon jetzt von qualifizierten Personen aus den anderen Dienstarten übernommen werden.

So werden Aufgaben wie venöse Blutentnahmen, intravenöse Injektionen, Maßnahmen des Wundmanagements, Diabetesberatung und -einstellung vielerorts dauerhaft von examinierten Pflegefachkräften erledigt. Darüber hinaus ist es häufig so, dass Patienten in den Notaufnahmen erst alle notwendigen diagnostischen Maßnahmen ohne ausdrückliche Anordnung erhalten, bevor sie einem Arzt vorgestellt werden. Entsprechende Verfahrensanweisungen, Weiterbildungen und Befähigungsnachweise sichern die notwendige Qualität.
Die Durchführungsverantwortung liegt selbstverständlich bei der ausführenden Person. Die Anordnungsverantwortung hat der Arzt. Im Rahmen des Wundmanagements, der Diabetesberatung und -einstellung nehmen die Ärzte diese Verantwortung nur pro forma wahr. Häufig werden die Empfehlungen der entsprechend zertifizierten Mitarbeiter ,ungesehen“ umgesetzt.

Die Bearbeitung von Modellprojekten beginnt eher schleppend, weil Kostenträger und Leistungserbringer gemeinsam diese Projekte definieren müssen. Die rechtssichere Durchführung der Modellprojekte ist Voraussetzung. Es müssen Kooperationspartner im Bildungssektor beteiligt werden, um die Inhalte der Modellprojekte in den einzelnen Curricula zu verankern.

Das notwendige Fazit der Modellphase kann nur eine gesetzliche Regelung sein, die Aufgaben aus der Heilkunde definiert, welche dauerhaft auf entsprechend ausgebildete Gesundheits- und Krankenpflegerinnen übertragen werden.

A. Koeppe $(\bowtie)$

Sana Ohre- Klinikum Haldensleben,

Haldensleben, Deutschland 